\title{
Monitoring adherence to guidelines of antibiotic use in pediatric pneumonia: the MAREA study
}

\author{
Pasquale Di Pietro ${ }^{1}$, Ornella Della Casa Alberighi ${ }^{\wedge}$, Michela Silvestri ${ }^{3}$, Maria Angela Tosca ${ }^{3}$, Anna Ruocco ${ }^{4}$, \\ Giorgio Conforti ${ }^{4}$, Giovanni A. Rossi ${ }^{3}$, Elio Castagnola ${ }^{5}$, Maria Caterina Merlano ${ }^{6}$, Simona Zappettini ${ }^{6}$, \\ Salvatore Renna ${ }^{1 *}$ and the Pediatric Ligurian Network MAREA network
}

\begin{abstract}
Background: Children are the most vulnerable population exposed to the use of antibiotics often incorrectly prescribed for the treatment of infections really due to viruses rather than to bacteria. We designed the MAREA study which consisted of two different studies: i) a surveillance study to monitor the safety/efficacy of the antibiotics for the treatment of pneumonia (CAP), pharyngotonsillitis and acute otitis media in children younger than 14 yrs old, living in Liguria, North-West Italy and ii) a pre-/post-interventional study to evaluate the appropriateness of antibiotic prescription for the treatment these infections. In this paper, we show only results of the appropriateness study about the antibiotic prescription for the treatment of pneumonia.

Methods: Patients included in this study met the following inclusion criteria: i) admission to the Emergency/ Inpatient Dpt/outpatient clinic of primary care pediatricians for pneumonia requiring antibiotics, ii) informed written consent. The practice of prescribing antibiotics was evaluated before-and-after a 1 day-educational intervention on International/National recommendations.

Results: Global adherence to guidelines was fulfilled in 45\%: main reason for discordance was duration (shorter than recommended). Macrolide monotherapy and cephalosporins were highly prescribed; ampicillin/amoxicillin use was limited. $61 \%$ of patients received $>1$ antibiotic; parenteral route was used in 33\%. After intervention, i) in all CAP, cephalosporin prescription decreased $(-23 \%)$ and the inappropriate macrolide prescriptions was halved and, ii) in not hospitalized CAP (notH-CAP), macrolides were prescribed less frequently $(-25 \%)$ and global adherence to guidelines improved (+39\%); and iii) in H-CAP antibiotic choice appropriateness increase.
\end{abstract}

Conclusion: Prescribing practices were sufficiently appropriate but widespread preference for multidrug empirical regimens or macrolide in monotherapy deserve closer investigation.

Keywords: Antibiotic therapy, Pneumonia, Children, Appropriateness

\section{Background}

Drug prescribing attitude varies from country to country probably related to the needs of different patient populations and to the individual attitudes of decision making among physicians [1]. Antibiotics are one of the most prescribed drugs throughout the world and their use appears to be significantly conditioned by patients' demand

\footnotetext{
*Correspondence: salvatore.renna@gaslini.org

Deceased

${ }^{1}$ Pediatric Emergency Department, Istituto G, Gaslini, Genoa, Italy Full list of author information is available at the end of the article
}

and by other social factors $[1,2]$. In Western countries, the most frequent indications for the prescription of antibiotics are upper and lower respiratory tract infections such as pneumonia both in adults and in children [3]. Unguided use can lead to drug wastage, lost in confidence in efficacious antibiotics, superinfection, ineffective treatment, delayed healing, unnecessary dependency from medical aid, spread of resistant organisms [4-6]. Among developed countries, Italy has one of the highest rates of antibiotic use [7]. Despite availability of guidelines also for the treatment of pediatric pneumonia [5, 8], overprescription/abuse 
of antibiotics in the treatment of respiratory infections is still a worldwide problem $[9,10]$. Also the Regional Health Plan of Liguria, promotes the reduction of the use of antibiotics and especially of last generation antibiotics when appropriate therapies equally effective against the etiologic agents are available. Educational interventions seem to be effective in improving a more rationale use of antibiotics [11-14]. Children are the most vulnerable population exposed to the use of antibiotics often incorrectly prescribed for the treatment of infections such as pneumonia, we designed the MAREA study (farMAcovigilanza in pediatria REgione LiguriA i.e. pharmacolovigilance in pediatric patients in Ligurian Region): one of the aim of this study was to evaluate the appropriateness of antibiotic prescription for the treatment of pneumonia before and after an educational intervention. The findings of this study may further confirm that priority actions for improving the rational use of antibacterials in children should concentrate on education activities such as active participation of the pediatricians to training courses.

\section{Methods}

\section{Study design}

The MAREA study comprised two different studies with different designs: i) a phase IV, time-cohort, multicenter 2 yr-surveillance study to monitor the safety/efficacy of the antibiotics for the treatment of pneumonia, pharyngotonsillitis and AOM in Liguria (Italy) and ii) a pre -/post-interventional study to evaluate the appropriateness of antibiotic prescription for the treatment these infections.

In this paper, we show only results of the appropriateness study about the antibiotic prescription for the treatment of pneumonia.

Briefly, for the surveillance study, patients were followed-up for 21 days with the aim to evaluate the frequency of i) hospitalization for pneumonia, pharyngotonsillitis or AOM, ii) the occurrence of adverse drug reactions (ADR), iii) the hospitalization for $A D R /$ serious adverse events.

The appropriateness study was designed to collect data on the disease management and antibiotic prescriptions and to evaluate possible effect of an educational intervention to increase appropriate management and antibiotic prescriptions.

A web-based electronic case report form (e-CRF) was used to collect all data anonymously. Briefly, after having obtained written informed consent by parents/tutors, each investigator uploaded data in a web-based e-CRF. To access the e-CRF, each investigator was requested to enter her/his login/password. Private data (such as first and last name or date of birth) were automatically encrypted before sending to the central server (so data were anonymously collected).
If during the follow-up, a patient needed to be visited by a physician different from the "enrolling" physician, data entered at the enrollment was accessible also to the "follow-up" physician who could update the e-CRF with additional information. Demographic (age and gender), antropometric (i.e. weight) and clinical data (i.e. diagnostic procedure and details of antibiotic administration) were recovered.

The surveillance and the appropriateness studies were approved by Ethics Committee of the Gaslini Institute. For the surveillance study, all participants and their parents or tutors were informed in detail on the experimental procedure and the main scope of the study and provided written informed consent: For the appropriateness study, in the pre-intervention phase, pediatricians were kept unaware of the aim of the study.

\section{MAREA patient population and setting}

Seventeen pediatric physicians (9 hospital physicians and 8 primary care pediatricians) belonging to the MAREA Network participated in the study and enrolled patients with the following characteristics: i) aged $<14$ yrs old, male and female; ii) patients admitted to the Emergency/ Inpatient Dpt or in outpatient clinic of family physicians with a diagnosis of pneumonia, pharyngotonsillitis or AOM requiring antibiotic treatment and iii) informed written consent. Exclusion criteria were as follows: cystic fibrosis, cancer, immune deficiencies, living outside Liguria.

Patients were enrolled during two periods (each of 11 months from February to December) (phase I: 2013 and phase II: 2014): between the two phases a 1-day educational intervention on the diagnosis and antibiotic treatment of respiratory tract infections was performed.

In this paper, we show only data on patients enrolled with a diagnosis of pneumonia.

\section{Training between phase I and II of the study}

This intervention consisted of 1-day educational sessions on the diagnosis and treatment of each of the three indications according to guidelines (Table 1). Briefly, all 17 physicians were exposed to the education intervention (all in one place). Some members of the MAREA Steering Committee ( 2 pediatric pulmonologists, 1 pediatric infectious disease specialist, 2 pediatric and medical emergency specialist and, 2 primary care pediatricians) provided educational sessions for the diagnosis and the treatment of pneumonia with specific recommendations for the best prescribing practices (International/National recommendations). On this occasion, the results of the first phase of the study were reported. Of note that in the first phase of this study, pediatricians were kept unaware of the evaluation, so their prescribing of antibiotic practices could not have been influenced by the study. Each session was 
Table 1 Drug treatments for pneumonia in children as suggested by Esposito S et al. (Esposito et al., 2012), revised

\begin{tabular}{|c|c|c|}
\hline Antibiotic choice & Dose and route of administration & Total duration of treatment \\
\hline \multicolumn{3}{|l|}{ First choice } \\
\hline Amoxicillin & $50-90 \mathrm{mg} / \mathrm{kg} / \mathrm{d}(\max 3.000 \mathrm{mg}$ ) in 2-3 doses. Oral & $5-7$ days \\
\hline Ampicillin & $100 \mathrm{mg} / \mathrm{kg} / \mathrm{d}$ (max $12.000 \mathrm{mg}$ ) in 3-4 doses. iv & $5-7$ days \\
\hline Clarithromycin & $15 \mathrm{mg} / \mathrm{kg} / \mathrm{d}$ (max $1.000 \mathrm{mg}$ ) in 2 divided doses. Oral & 14 days \\
\hline Clarithromycin & $4-8 \mathrm{mg} / \mathrm{kg} /$ day in 2 divided doses. iv & 14 days \\
\hline \multirow[t]{2}{*}{ Azithromycin } & $10 \mathrm{mg} / \mathrm{kg} / \mathrm{d}$ (max $500 \mathrm{mg}$ ) in 1 dose. Oral & 3 days \\
\hline & 1 dose of $10 \mathrm{mg} / \mathrm{kg} / \mathrm{d}$ and then $5 \mathrm{mg} / \mathrm{kg} / \mathrm{d}$. oral & 4 days \\
\hline \multicolumn{3}{|l|}{ Second choice } \\
\hline Amoxicillin/clavulanate & $\begin{array}{l}\text { amoxicillin component: 50-90 mg/kg/d (max } 3.000 \text { mg) } \\
\text { in } 2 \text { doses. Oral }\end{array}$ & 5-7 days \\
\hline Ampicillin/sulbactam & $150 \mathrm{mg} / \mathrm{kg} / \mathrm{d}$ (max $9.000 \mathrm{mg}$ ) in 3 doses. iv & \\
\hline Cefuroxime axetil & 20-30 mg/kg/d in 2 divided doses. Oral & \\
\hline Benzylpenicillin iv & 200.000 units $/ \mathrm{kg} / \mathrm{d}$ in $4-6$ doses. iv & \\
\hline Ceftriaxone & 80-100 mg/kg (max $2.000 \mathrm{mg}$ ) once a day. iv & \\
\hline Cefotaxime & $100-150 \mathrm{mg} / \mathrm{kg} / \mathrm{d}$ in 3 divided doses. iv & \\
\hline Cefaclor & 20-40 mg/kg once a day. Oral & \\
\hline Cefpodoxime proxetil & $8 \mathrm{mg} / \mathrm{kg}$ once a day. Oral & \\
\hline
\end{tabular}

Combination therapy with a beta-lactam drug and a macrolide can be considered in more severe cases or when an atypical bacterial infection is suspected

followed by a $15-30$ min discussion of the guideline indications and of the results obtained. In addition, each physician received a copy of the slides that were shown and concise written materials with focused guidance on pneumonia. The second phase took place immediately after the educational intervention. Monthly update about the enrolment rate in the second phase was sent to the physician.

\section{Definition of pneumonia}

According to the clinical findings, children were diagnosed as pneumonia if they had recurrent or persistent fever $\left(>38.5{ }^{\circ} \mathrm{C}\right)$ associated to chest indrawing and increased respiratory rate [5]. Pneumonia was confirmed by chest X-rays. (that were performed in almost all patients hospitalized children i.e. in 135 out of 137).

\section{Criteria for admission}

The appropriateness of the disease management i.e. for example the decision of hospitalizing patients, will be reported in a future paper. Briefly, the following clinical and demographic characteristics were collected: i) respiratory difficulty, ii) local hypophonesis/localized crackles, iii) recurrent/persistent body temperature $\geq 38.5^{\circ} \mathrm{C}$, iv), dehydration, v) thoracic and/or abdominal pain, vi) $\mathrm{O}_{2}$ saturation $<92 \%$ (in children aged $<5$ yrs. old, $<95 \%$ ) vii) tachidyspnoea, viii) persistent dry cough, ix) nasal flaring, $\mathrm{x}$ ) cyanosis, xi) comorbidities, xii) age $\leq 1$ yr. old, xiii) inability to feed her/himself, xiv) poor familiar compliance.

\section{Antibiotic prescription}

The practice of prescribing antibiotics was evaluate before and after a 1 day-educational intervention on the diagnosis and antibiotic treatment of respiratory tract infections in children based on International/National recommendations $[5,8]$ (Table 1 )

The appropriateness of the antibiotic prescriptions was assessed by comparing the clinical practice of each physician with consensus guidelines developed for pneumonia and presented at the educational sessions. The following aspects were assessed: i) indication-appropriate decision making regarding use/ nonuse of antibiotics; ii) antibiotic choice; iii) total duration of antibiotic use; iv) dose. We considered as appropriate a choice of an antibiotic independently if it was a "first" or a "second" choice.

The global adherence of physicians' antibiotics prescription was considered appropriate when totally according to the current clinical practice guidelines and any divergence from the guidelines led to a final assessment of the treatment course as discordant with the guidelines.

Each parameters was also evaluated separately so that the nonadherence to one parameter or missing data for one parameter did not preclude assessment of the others. If more than 1 drug was prescribed, total 
duration of antibiotic use and dose were evaluated separately for each drug.

Appropriateness of prescriptions was expressed as percentage.

\section{Statistical analysis}

The needed sample size was calculated, assuming an expected frequency of hospitalization of about 10\% in pediatric population with a diagnosis of pneumonia, pharyngotonsillitis and/or AOM, the type I error $=0.05$, the power $=0.80$ and the exact binomial test. With these assumptions, the needed sample size ranges was 840 patients (420 patients each phase). Being the appropriateness study strictly depend on the surveillance study (i.e. the number antibiotics prescriptions are related to the number of patients enrolled), the same sample size was kept also for the appropriateness study. The needed sample size and power was calculated according to Gatsonis $\mathrm{C}$ and Sampson AR formulations and using the Software NQuery Advisor (release 7.0).

Descriptive statistics of the characteristics of the patients were performed and reported in terms of mean and standard deviation (SD) for the quantitative variable i.e. chronological age and in terms of absolute frequencies and percentages for the qualitative variables (i.e. antibiotic prescriptions).

Comparison of frequency data was performed by the chi-square test or by the Fisher's exact test in case of expected frequencies less than five. All tests were twosided and a $P$ value less than 0.05 was considered statistically significant. "Statistica release 6" (StatSoft Corp., Tulsa, OK, USA) was used.

\section{Results}

Analyzing the whole MAREA population (i.e. those with pneumonia, pharyngotonsillitis and/or AOM), we found a higher frequency of hospitalization for the three indications (i.e pneumonia, pharyngotonsillitis or AOM) than expected ( $25 \%$ vs. $10 \%)$.

Demographic characteristics of the study population $(N=225)$ are reported in Table 2.
Overall, a high number of patients with pneumonia were severe enough to require hospital admission (No. 137, $60.9 \%$ ): $66.2 \%$ phase I and $51.8 \%$ in phase II.

\section{Antibiotic prescription before-and-after educational intervention in the whole population of children with pneumonia and in hospitalized (H-CAP) and not-hospitalized pneumonia (NotH-CAP)}

In all, 225 children enrolled for pneumonia received a total of 400 antibiotic prescriptions. Distribution of prescriptions by antibiotic therapeutic category (aminopenicillins, cephalosporines and macrolides) and by antibiotic molecule are reported in Fig. 1. Aminopenicillins and macrolides were the most frequently used class of antibiotics (Fig. 1a) both in phase I (Fig. 1b) and in phase II (Fig. 1c). Cephalosporins were prescribed in $11.5 \%$ (46 prescriptions) of the patients (Fig. 1a), 23\% lower in phase II (phase I: 31 prescriptions, 12.6\%, phase II: 15 prescriptions, 9.7\%, $p=.38$ ) (Fig. $1 \mathrm{~b}$ and c).

Clarithromycin (160 prescriptions, 40.0\%) followed by ampicillin + enzyme inhibitor (EI) (96 prescriptions, $24.0 \%$ ) or amoxicillin + enzyme inhibitor (EI) (85 prescriptions, 21.3\%) were the most frequently prescribed drugs (Fig. 1a). Ampicillin/amoxicillin (without EI) were prescribed only in 4 cases (Fig. 1a).

About $61 \%$ of children (No. 138) received more than one antibiotic: the most frequent prescriptions was macrolide and aminopenicillins + EI (No. 111/138, 80.4\%) both in phase I (No. 60/76, 79.0\%) and in phase II (No. 51/62, $82.3 \%$ ). About $50 \%$ of children who received more than one antibiotic was treated with a combination of macrolide and ampicillin + EI (No. 73/138, 52.9\%): this combination was prescribed about 7-fold more frequently in H-CAP children (No. 69/99, 69.70\%) than in notH-CAP children (No. 4/39, 10.26\%) $(p<.0001)$. In addition, macrolide was more frequently prescribed with ampicillin + EI in H-CAP (69/99 prescriptions, $69.70 \%)$ and to amoxicillin + EI in notH- CAP (34/39 prescriptions, $87.18 \%$ ).

Macrolide monotherapy was prescribed in 36 cases ( $9 \%$ of total prescriptions).

Comparing H-CAP and notH-CAP, we found a statistically significant difference in the distribution of antibiotic

Table 2 Demographic and clinical characteristics of the study population

\begin{tabular}{llll}
\hline & $\begin{array}{l}\text { All } \\
\text { (No.225) }\end{array}$ & $\begin{array}{l}\text { Hospitalized pneumonia } \\
(\text { No.137) }\end{array}$ & $\begin{array}{l}\text { Not-hospitalized pneumonia } \\
\text { (No.88) }\end{array}$ \\
\hline Age [months (mean (SD)] & $60.73(34.03)$ & $55.99(32.00)$ & $68.06(35.93)$ \\
Gender (male-to-female ratio) & 1.06 & 0.89 & 1.38 \\
Enrollment in phase I [No. (\%)] & $142(63.11)$ & $94 / 142(66.20)$ & $48 / 142(33.80)$ \\
& & $94 / 137(68.61)$ & $48 / 88(54.55)$ \\
Enrollment in phase II [No. (\%)] & $83(36.89)$ & $43 / 83(51.81)$ & $40 / 83(48.19)$ \\
& & $43 / 137(31.38)$ & $40 / 88(45.45)$ \\
\hline
\end{tabular}




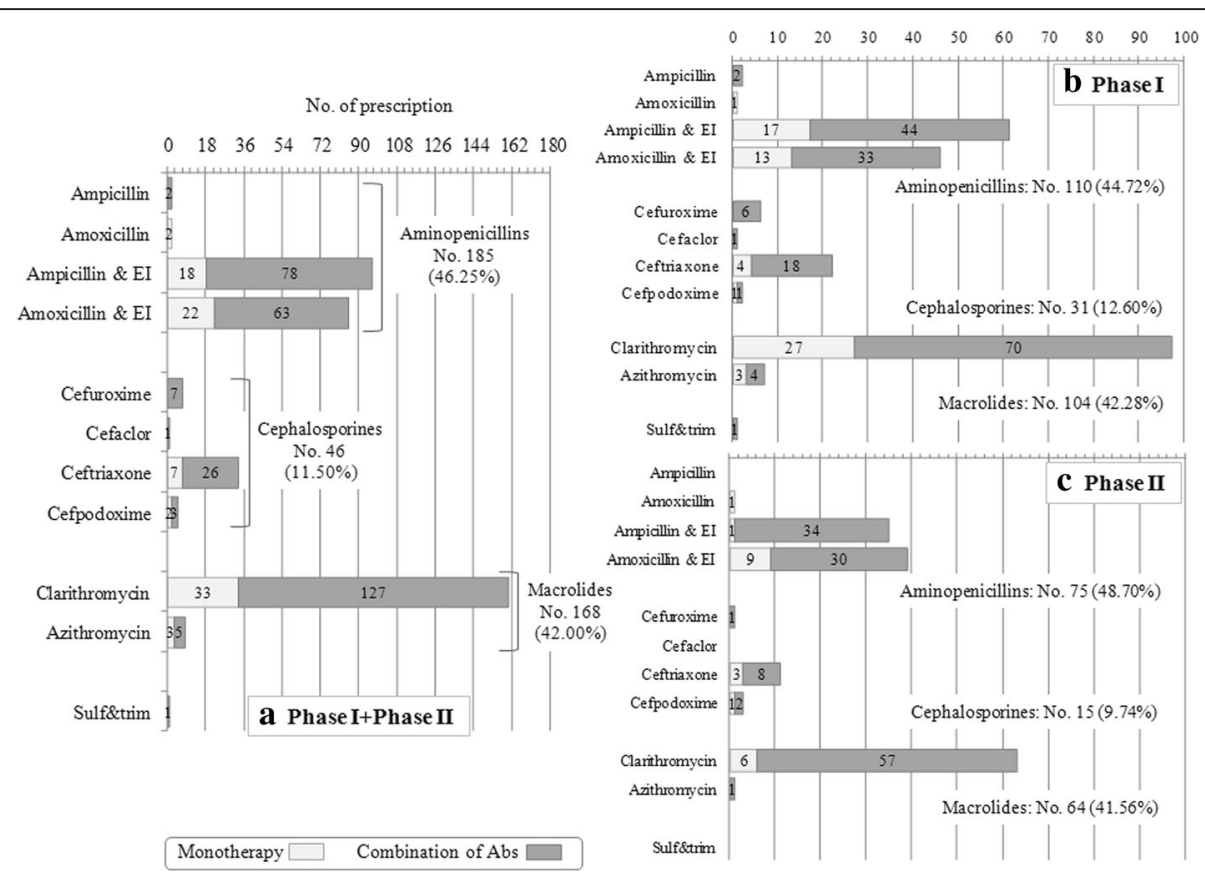

Fig. 1 Antibiotic prescription for the treatment of pneumonia in all the studied period (panel a) or before (phase l, panel b) and after 1 day-educational intervention (phase II, panel c). Type of antibiotics is reported on the ordinate and the number of prescription on the abscissa

therapeutic category prescriptions $(p=.003)$ : cephalosporins were prescribed 5 -fold more frequently in $\mathrm{H}$ CAP (Fig. 2a) than in notH-CAP (Fig. 2b) being $15.30 \%$ (41/268 prescriptions) vs $3.79 \%$ (5/132 prescriptions), respectively: H-CAP patients were more likely to receive cephalosporins as compared to notH-CAP
(OR: 4.59 [95\%CI: 1.77-11.90], $p=.0007)$. Differently, the frequency of prescriptions of aminopenicillins and macrolides were similar in the two groups (Fig. 2). Analyzing the distribution of prescriptions by antibiotic molecule, we found that ampicillin + EI $(p<.001)$ and ceftriaxone $(p=.0001)$ were administered significantly

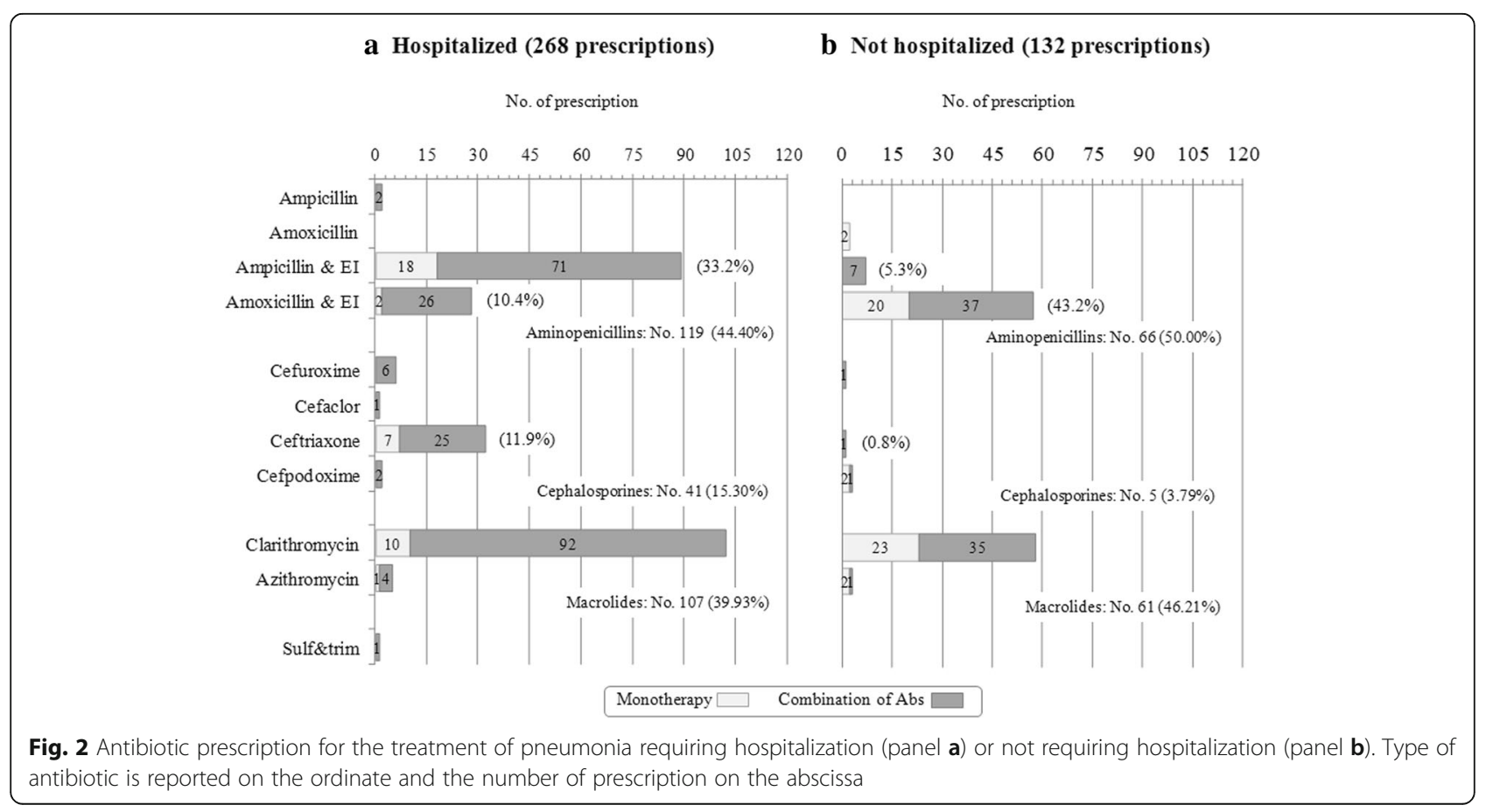


more frequently in H-CAP (Fig. 2a) whereas amoxicillin + $\mathrm{EI}(p<.001)$ more frequently in notH-CAP (Fig. $2 \mathrm{~b})$.

A combination of antibiotics was used more frequently to treat H-CAP patients $(72.26 \%, 99 / 137)$ than notHCAP children $(44.32 \%, 39 / 88)(p=.0007)$.

In $\mathrm{H}$-CAP, no difference in the prescription of each class of antibiotics was seen between phase I and phase II, (Fig. 3a and b). In notH-CAP, educational intervention was followed by a $25 \%$ decrease in macrolide prescription (phase I: $34 / 64$ prescriptions, $53.13 \%$ vs. phase II: $27 / 68$ prescriptions, $39.71 \%, p=.12$ ) (Fig. $3 \mathrm{c}$ and d).

About two-third of prescriptions were for oral use (268/400 prescriptions), both in phase I and in phase II (Table 3), especially macrolides (268/400 prescriptions, $62.31 \%)$ followed by ampicillin/amoxicillin + EI (87/400 prescriptions, $32.46 \%$ ).

The parenteral route accounted for $33.00 \%$ of prescriptions (Table 3), and it was more commonly use to administer antibiotics to H-CAP (46.27\%) than to notH-CAP (6.06\%) children $(p<.0001)$ (Table 3$)$; among parenteral antibiotic, ampicillin alone or added to sulbactam was the most frequently administered (98 prescriptions, $74.24 \%$ of all parenteral prescriptions), followed by third-generation cephalosporins (33 prescriptions, 25.00\%).

Patients were treated by parenteral antibiotics for a mean of 6.28 (SD: 3.77) days, a longer time of treatment if they were hospitalized for pneumonia [6.66 (SD:3.66) days] than if they had milder pneumonia [1.75 (SD: 1.49) days] $(p=.001)$. In phase II, a slight decrease in parenteral antibiotic prescription was observed but without reaching statistically significance $(34.96 \%$ vs. $29.87 \%$, respectively, $p=.29$ ).

\section{Adherence to guidelines: Assessment of individual parameters}

Parameters were evaluated separately, so that nonadherence to one parameter did not preclude assessment of the others.

\section{Indication and antibiotic choice}

The decision making regarding use or nonuse of antibiotics (i.e. indication) was appropriate in all cases.

Antibiotic choice was adherent to the recommendations in $92.50 \%$ of the cases without statistically significant difference between phase I and phase II $(p=.17)$ (Table 4). Adherence to guidelines was higher for $\mathrm{H}$ CAP than for not-H-CAP $(96.27 \%$ vs. $84.85 \%$; $p<.001)$ (Table 5); this was true both in phase 1 (H-CAP: 172/ 182 prescriptions, $94.51 \%$ vs. not-H-CAP: $52 / 64$ prescriptions, $81.25 \% ; p=.001$ ) and in phase 2 (H-CAP: 86/ 86 prescriptions, $100 \%$ vs. not-H-CAP: $60 / 68$ prescriptions, 88.24\%; $p=.001$ ). In addition, in H-CAP, we found a significant increase after the educational intervention and the adherence to guidelines reached $100 \%(p=.03)$ (Table 6). Differently, in notH-CAP, appropriateness rate for antibiotic choice remained almost stable in phase II (phase I: $81.25 \%$, phase II: $88.24 \%, p=.26$ ) (Table 6).

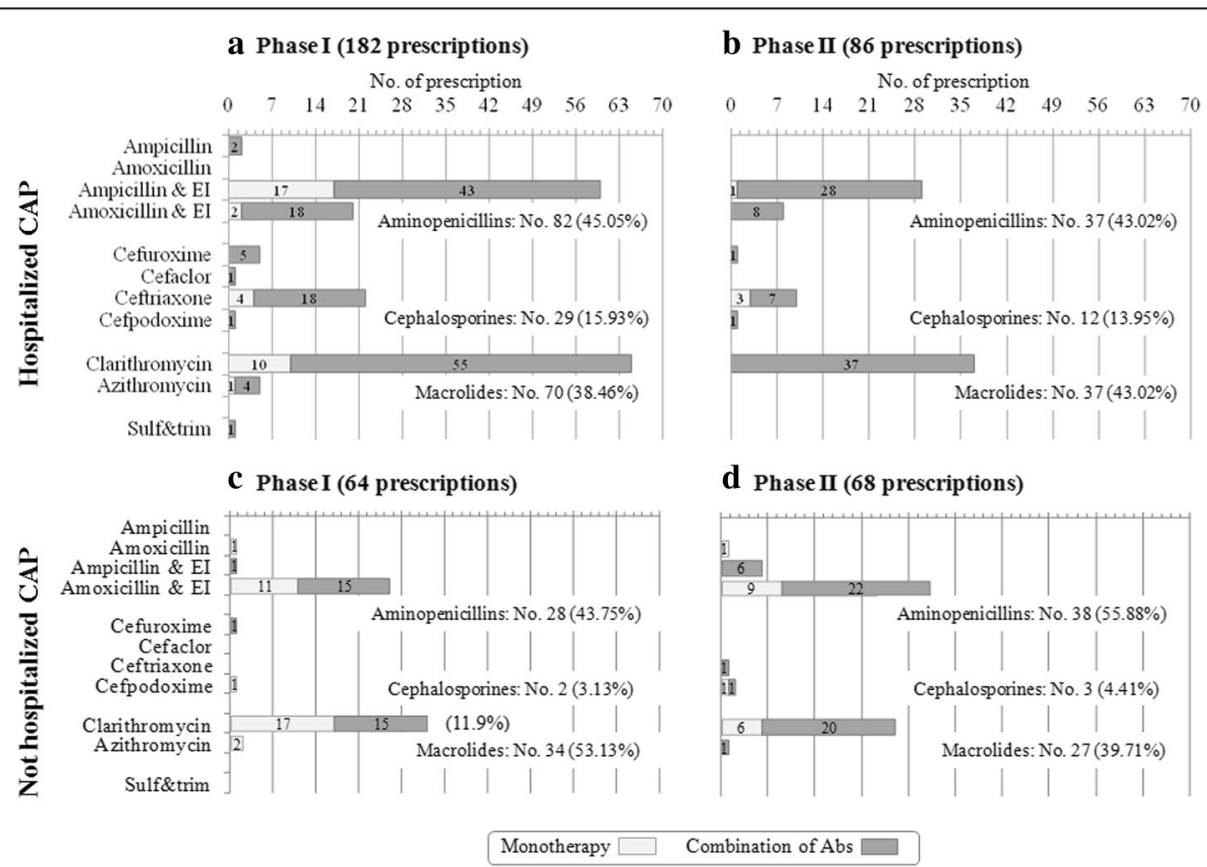

Fig. 3 Antibiotic prescription for the treatment of pneumonia requiring hospitalization (panels $\mathbf{a}$ and $\mathbf{b}$ ) or not requiring hospitalization (panels $\mathbf{c}$ and $\mathbf{d}$ ) before (panels $\mathbf{a}$ and $\mathbf{b}$ ) and after (panels $\mathbf{b}$ and $\mathbf{d}$ ) 1 day-educational intervention. Type of antibiotics is reported on the ordinate and the number of prescription on the abscissa 
Table 3 Route of administration for antibiotics in hospitalized (H-CAP) and not-hospitalized (notH-CAP) patients before and after educational course

\begin{tabular}{|c|c|c|c|c|}
\hline & \multicolumn{2}{|c|}{ Route of administration for antibiotics } & \multicolumn{2}{|l|}{$P$ value } \\
\hline & $\begin{array}{l}\text { Oral } \\
\text { [No (\%)] }\end{array}$ & $\begin{array}{l}\text { Parenteral } \\
{[\text { No (\%)] }}\end{array}$ & Before vs. after & H-CAP vs. notH-CAP \\
\hline Prescriptions for the whole study population (No. 400) & $268(67.00)$ & $132(33.00 \%)$ & & \\
\hline Before educational course (No. 246) & $160(65.04)$ & $86(34.96)$ & 0.29 & \\
\hline After educational course (No. 154) & $108(70.13)$ & $46(29.87)$ & & \\
\hline Prescriptions for hospitalized patients (No. 268) & $144(53.73)$ & $124(46.27)$ & & $<.0001$ \\
\hline Before educational course (No. 182) & $97(53.30)$ & $85(46.70)$ & 0.84 & $<.001$ \\
\hline After educational course (No. 86) & $47(54.65)$ & $39(45.35)$ & & $<.001$ \\
\hline Prescriptions for not-hospitalized patients (No. 132) & $124(93.94)$ & $8(6.06)$ & & \\
\hline Before educational course (No. 64) & $63(98.44)$ & $1(1.56)$ & 0.06 & \\
\hline After educational course (No.68) & $61(89.71)$ & $7(10.29)$ & & \\
\hline
\end{tabular}

Comparing the frequency of inappropriateness in antibiotic choice for the different antibiotic classes, we found that in phase I the frequency of inappropriate prescriptions was higher for macrolides than for other antibiotics (i.e. aminopenicillins or cephalosporines) (14/104 prescriptions vs. $8 / 142$ prescriptions, $13.46 \%$ vs $5.63 \%$, respectively, $p=.034$ ). In phase II, the frequency of inappropriate prescriptions was further reduced and became similar being $7.81 \%$ (5/64 prescriptions) for macrolides and 3.33\% (3/90 prescriptions) for other antibiotics $(p=.22)$. This was mainly due to a $45 \%$ reduction in inappropriate macrolide prescription in phase II even though not statistically significant $(p=.26$ ), (data not shown).

\section{Total duration of antibiotic use}

In $62.21 \%$ of prescriptions, total duration of antibiotic use was concordant with the guidelines; educational intervention only slightly increase adherence $(p=.41)$ (Table 4). Inappropriate duration was seen only for macrolides that were used shorter than recommended [mean duration (days) 9.65 (SD: 1.76); min:3, max: 13]: the mean duration of antimicrobial treatment with macrolides was 9.31 (SD 3.04) days in hospitalized and
9.83 (SD 2.49) days in not-hospitalized patients ( $p=0.27$ ). In notH-CAP, a 36\% increase in guideline adherence of duration of treatment was observed in phase II $(p=.025)$ (Table 6).

Analyzing only macrolide prescriptions appropriate for choice, we found that only $13.19 \%$ of them were concordant to the guidelines (19/144 prescriptions) also for duration with an about 30\% increase in adherence in phase II but without reaching statistically significance (phase I: $10 / 85$ prescriptions, $11.76 \%$, phase II: $9 / 59$ prescriptions, $15.25 \%, p=.54)$. In notH-CAP, a 5 -fold increase in adherence also for duration was found after the educational intervention being $4.76 \%$ (1/21 prescriptions) before and $27.27 \%$ (6/22 prescriptions) after but this enhancement was not statistically significant $(p=.095)$.

\section{Dose}

The dose of antibiotics was concordant with the guidelines in $81.20 \%$ (324 prescriptions) of the 399 evaluated doses ( 1 case missing) (Table 4). In the discordant doses (75 prescriptions), the dose per kilogram was lower than that recommended in $66.67 \%$. Educational intervention did not modify the appropriateness rate for this

Table 4 Frequency of appropriateness of antibiotic use in children with pneumonia evaluated before and after educational course

\begin{tabular}{|c|c|c|c|c|}
\hline & $\begin{array}{l}\text { Children with pneumonia (phase I + II) } \\
\text { (No. } 400 \text { prescriptions) }\end{array}$ & $\begin{array}{l}\text { Pre-educational course (phase I) } \\
\text { (No. } 246 \text { prescriptions) }\end{array}$ & $\begin{array}{l}\text { Post-educational course (phase II) } \\
\text { (No. } 154 \text { prescriptions) }\end{array}$ & $\begin{array}{l}P \\
\text { value }\end{array}$ \\
\hline \multicolumn{5}{|l|}{ Single parameter appropriateness } \\
\hline Indication [No. (\%)] & $400(100.00)$ & - & - & - \\
\hline Choice of antibiotic [No. (\%)] & $370(92.50)$ & $224(91.06)$ & $146(94.81)$ & .17 \\
\hline Duration [No. (\%)] & $242 / 389^{a}(62.21)$ & $143 / 236^{\mathrm{a}}(60.59)$ & $99 / 153^{\mathrm{a}}(64.71)$ & .41 \\
\hline Dose [No. (\%)] & $324 / 399^{\mathrm{a}}(81.20)$ & $198 / 245^{\mathrm{a}}(80.82)$ & $126(81.82)$ & .80 \\
\hline $\begin{array}{l}\text { Global appropriateness [No. } \\
(\%)]\end{array}$ & $175 / 389^{\mathrm{a}}(44.99)$ & $103 / 236(43.64)$ & 72/153 (47.06) & .51 \\
\hline
\end{tabular}

${ }^{a}$ when data are lacking, the total number of data available are reported 
Table 5 Frequency of appropriateness of antibiotic use in children with pneumonia requiring or not requiring hospitalization

\begin{tabular}{|c|c|c|c|c|}
\hline & $\begin{array}{l}\text { Children with pneumonia } \\
\text { (No. } 400 \text { prescriptions) }\end{array}$ & $\begin{array}{l}\text { Hospitalized CAP } \\
\text { (No. } 268 \text { prescriptions) }\end{array}$ & $\begin{array}{l}\text { Not hospitalized CAP } \\
\text { (No. } 132 \text { prescriptions) }\end{array}$ & $P$ value \\
\hline \multicolumn{5}{|l|}{ Single parameter appropriateness } \\
\hline Indication [No. (\%)] & $400(100.00)$ & - & - & - \\
\hline Choice of antibiotic [No. (\%)] & $370(92.50)$ & $258(96.27)$ & $112(84.85)$ & $<.001$ \\
\hline Duration [No. (\%)] & $242 / 389^{a}(62.21)$ & $162 / 261^{\mathrm{a}}(62.07)$ & $80 / 128^{\mathrm{a}}(62.50)$ & .93 \\
\hline Dose [No. (\%)] & $324 / 399^{a}(81.20)$ & $235 / 267^{\mathrm{a}}(88.01)$ & $89(67.42)$ & $<.001$ \\
\hline Global appropriateness [No. (\%)] & $175 / 389^{\mathrm{a}}(44.99)$ & 132/261 (50.57) & 43/128 (33.59) & .0016 \\
\hline
\end{tabular}

${ }^{a}$ when data are lacking, the total number of data available are reported

parameter (Table 4) both in H-CAP (Table 6) and in notH-CAP (Table 6).

Concordant doses were found more frequently in $\mathrm{H}$ CAP than in notH-CAP being $88.01 \%$ and $67.42 \%$, respectively $(p<.001)$ (Table 5$)$; this was true both in phase I $(p=.0003)$ and in phase II $(p=.0003)$ (data not shown).

\section{Global adherence to guidelines}

Global adherence to guidelines for all parameters was fulfilled in $45 \%$ of the cases. Age did not affect the frequency of concordant prescriptions (not shown). No statistically significant difference between phase I and phase II was found both in all CAP and in H-CAP (Tables 4 and 6). Differently, in notH-CAP, the educational intervention determined a $39.26 \%$ improvement in global adherence to guidelines without reaching statistically significance (phase I: $27.87 \%$, phase II: $38.81 \%, p=.19$ ) (Table 6).

Analyzing inappropriate prescriptions $(\mathrm{No}=220)$, the main reason for discordance was duration of treatment (147 prescriptions, $66.81 \%$ of all 220 discordant cases), followed by wrong dose (75 prescriptions, $34.09 \%$ of discordant cases) and antibiotic choice (30 prescriptions, $13.64 \%$ of discordant cases). Similar results were obtained analyzing the two phases separately (not shown).

Since the most common deviation from the guidelines was duration, we re-evaluated "global appropriateness" only considering concordance/discordance for indication, antibiotic choice and dose. We found an almost $60 \%$ of adherence to guidelines (235 prescriptions, $58.75 \%$ ). Again, in notH-CAP, after educational intervention, appropriateness significantly increased from $46.88 \%$ to $64.71 \%$ (30/64 prescriptions to $44 / 68$ prescriptions, respectively; $p=.039$ ).

No adverse event was reported.

\section{Discussion}

The main findings of this study are: i) the decision making regarding use/nonuse of antibiotics was appropriate in all cases but global adherence to guidelines was fulfilled in less than a half of the cases and the main reason for discordance was duration of treatment; ii) cephalosporins and macrolide monotherapy were highly prescribed whereas use of unprotected ampicillin/ amoxicillin was very limited; iii) a high percentage of patients received more than one antibiotic and parenteral route was used in over one third of prescriptions; iv) after educational intervention: inappropriate macrolide prescriptions was halved; in notH-CAP, cephalosporin and macrolide prescriptions decreased, global adherence to guidelines and appropriateness of duration of treatment improved; in H-CAP, antibiotic choice appropriateness rate increased.

Despite availability of guidelines, overprescription/ abuse of antibiotics in the treatment of respiratory infections is still a worldwide problem $[9,10]$. About $50 \%$ of antibacterial prescriptions for children are inappropriate $[15,16]$ and, in low-income countries, this percentage is even higher [17].

There are several reasons for these unjustified prescriptions: i) to be conditioned by parental pressure to receive antibiotics $[1,2,11,18]$; ii) the idea that antibiotic use can be more effective than diagnostic tests and observation of patient evolution [18] and may prevent bacterial superinfections and their complications [18-20]; iii) inertia related to previous practice patterns; iv) lack of awareness, inadequate knowledge, or perceived ineffectiveness of the guidelines [21].

In our studied population, the decision making regarding use/nonuse of antibiotics was appropriate in all cases. However, a rational drug use occurs not only when an appropriate drug is prescribed but also when it is administered in correct doses and for an adequate duration of therapy [22]. Several studies investigated not only the appropriateness of the choice of the molecule but also the duration of use and the dose, but all of them included adult population or patients with other diseases [23-25]. We evaluated a "global" adherence to guidelines of antibiotic use according to the document approved by the consensus conference for the treatment of respiratory tract infections in children which promoted not only the rational use of antibiotics, but also focusing on the appropriate agent, dosage and optimal duration of 


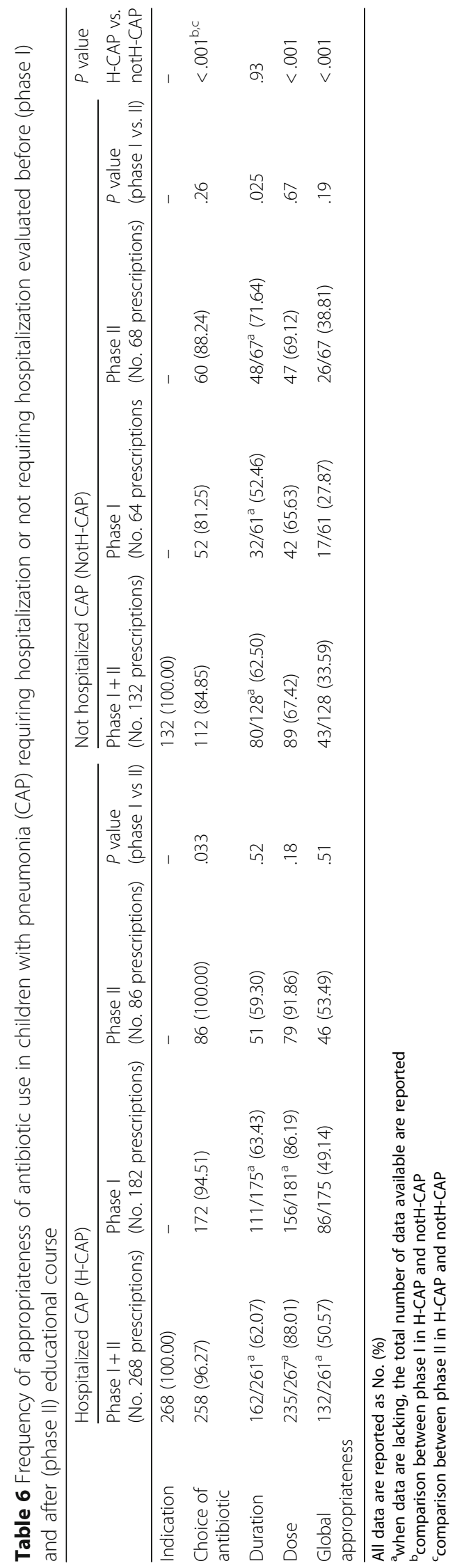


antibiotic therapy [8]. Before the educational training, adherence to guidelines in antibiotic use for all parameters was fulfilled in less than a half of the cases and the main reason for discordance was duration of treatment, that was shorter than recommended. Other healthcare settings in adult population reported similar results: adherence rates to guidelines of $44-49 \%$ have been reported in ambulatory patients with community-acquired pneumonia [26].

Assessing individual parameters, antibiotic choice was adherent to the recommendations in over $90 \%$ of the cases and was higher for H-CAP. In phase I, macrolides were inappropriately prescribed more frequently than other antibiotics (13.5\% vs $5.6 \%)$. In a retrospective analysis of 1 year-ambulatory pediatric visits, Saleh et al. [27] found similar results in children with pneumonia: macrolides were not recommended in $22.5 \%$ of cases. Similarly, Kronman et al. [28] found that the use of macrolides ranges from $27.8 \%$ to $41.7 \%$ of all antibiotics prescribed for childhood CAP and this percentage increased $10.0 \%$ every 2 years over the entire 14 year-study. The frequent use of macrolides should be discouraged because it can increase the burden of antibiotic resistance among pneumococci $[29,30]$. According to European surveillance data, the frequency of macrolide resistance among pneumococci isolates from Italy [29] is already the one of highest in Europe [31] being between 10\% and $29 \%$. In Italy, regardless the disease, cephalosporins are widely prescribed, while they represent less than $1 \%$ of the pediatric prescriptions in Denmark and the Netherlands [32]. Also our study confirms a relatively high cephalosporin use being prescribed in $11.5 \%$ of cases, predominantly in hospitalized patients (15\% vs $3 \%)$. This result together to the relatively high rates of macrolide monotherapy $(9 \%)$ and the very low rates of use of unprotected ampicillin $(0.75 \%$ in $\mathrm{H}-\mathrm{CAP})$ or amoxicillin use $(1.52 \%$ in notH-CAP) highlight a need for effective initiatives to improve the use of antimicrobial agents. Indeed, amoxicillin is considered the first-line antibiotic for the most common paediatric infectious diseases. In Italy, amoxicillin represents about one-fifth of antibiotic paediatric prescriptions, whereas in Canada and in the Netherlands, it represents half of the prescriptions [33]. In Italy, amoxicillin/clavulanate prescriptions are 3-fold higher than in the Netherlands and 5-fold higher than in Canada [33] and the trend is increasing. The preference of amoxicillin/clavulanate is mainly due to the fear of infections caused by beta-lactamase-producing bacteria.

Of note that more than $60 \%$ of patients received more than one antibiotic similarly to the results reported by DeLuca et al. [34] who found that $50 \%$ of LRTI cases received more than one antibiotic. The most frequent prescriptions was macrolide and aminopenicillins + EI. This may be explained considering that even though the main cause of pneumonia is Streptococcus pneumonia (the resistance of Streptococcus pneumoniae could be overcome by high dose of ampicillin (up to $80 \mathrm{mg} / \mathrm{kg}$ ) rather than by adding of clavulanate), also pneumonia due to Mycoplasma pneumoniae is not very unusual in preschool children [35].

Despite the fact that there are no evidence supporting the superiority of intravenous/intramuscular treatment of pneumonia as compared to the oral one, in our study parenteral route accounted for about one third of prescriptions and among them ampicillin alone or added to sulbactam was the most frequently administered (74.2\% of all parenteral prescriptions), followed by third-generation cephalosporins (25.0\%). An abuse of parenteral cephalosporins in Italian hospitalized children was already denounced in a study conducted last decade by Esposito et al. [36]. Parenteral administration may be justified by the clinical conditions of the patients: oral absorption of drug may be disturbed due to vomiting or in severely ill patients.

We also reported that duration of use and dose of antibiotics was concordant with the guidelines in over $60 \%$ and in over $80 \%$, respectively. Inappropriate duration was seen only for macrolides that were used shorter than recommended (mean: 9.6 days. 9.3 days in hospitalized and 9.8 days in not-hospitalized patients). - the guidelines we refer suggested to use macrolides for 14 days but other recommendations such as guidelines by the Pediatric Infectious Diseases Society and the Infectious Diseases Society of America suggests to prescribe a shorter course of antibiotic treatment at least in milder pneumonia [37]. In our sample population of not hospitalized CAP, the duration of treatment was longer [9.8 (SD 2.5) days] than that suggested by Pediatric Infectious Diseases Society and the Infectious Diseases Society of America guidelines. In discordant doses, the dose per kilogram was lower than that recommended in $67.6 \%$ of the cases. Concordant doses were found significantly more frequently in H-CAP than in notHCAP being $88.01 \%$ and $67.42 \%$, respectively.

On the basis of these results, guidelines alone seem to have a limited efficacy in changing physician behavior in the management of pediatric pneumonia, probably because primary care pediatricians have been faced with a variety of Italian/international guidelines, sometimes with contrasting key messages, requiring substantial time and effort to be analyzed and followed. Therefore, paediatricians knowledge and approach to antibiotic use require corrections to improve prescribing practices $[11-14,38]$. Educational campaigns/interventions promoting the rational use of antibiotics have been demonstrated to be effective in improving physician and nurse practitioner behavior [11-14] and particularly, if providing concise written materials with focused guidance on specific diseases [11]. Neuman and colleagues [39] recently 
demonstrated that after an introduction of an antimicrobial stewardship program and a clinical practice guidelines, the use of ampicillin increased by $34 \%$, whereas ceftriaxone use was halved. Similarly, it has been reported a greater use of narrow-spectrum antibiotics, such as penicillin and aminopenicillins, among children admitted to hospitals with clinical practice guidelines as compared to those without guideline [39]. After educational intervention, we found a $23 \%$ decrease in cephalosporin prescriptions in all CAP and a $25 \%$ decrease in macrolide prescription in notH-CAP.

Global adherence to guidelines was similar in the two phases of the study. However, analyzing antibiotic prescriptions in notH-CAP, the educational intervention determined an about 44\% improvement in overall adherence to guidelines. Parameters were also evaluated separately, so that nonadherence to one parameter did not preclude assessment of the others. Antibiotic choice appropriateness rate was not modified by the educational intervention being very high already in phase I (91\%). However, analyzing only H-CAP, we found a significant increase in antibiotic choice appropriateness rate after the educational intervention and the adherence to guidelines reached $100 \%$. Particularly, macrolide prescriptions were prescribed inappropriately in $13.46 \%$ and this rate was halved after educational intervention.

Regarding the duration of use, educational intervention only slightly increase adherence to guidelines from $60.59 \%$ to $64.71 \%$. However, in notH-CAP, a $36 \%$ increase in adherence to guidelines was observed in phase II. Inappropriate duration was seen only for macrolides that were used shorter than recommended.

The dose of antibiotics was concordant with the guidelines in $81.20 \%$ of the 400 evaluated doses. In the discordant doses, the dose per kilogram was lower than that recommended in $67.57 \%$ of the cases. Concordant doses were found more frequently in H-CAP than in notH-CAP being $88.01 \%$ and $67.42 \%$. Educational intervention did not modify this rate.

In our study, no ADR was reported. The lack of compliance in reporting ADRs is one of the main weakness of the paediatric pharmacovigilance system [40]. Educational training on pharmacovigilance at the territorial level should be encouraged and may facilitate a better compliance and thus optimisation of ADRs reporting.

Even if involving a single Italian region (Liguria, Northern Italy), we regard the external validity of our findings to be high, since our data represent unselected and consecutive patients. However, due to a high heterogeneity of drug use among Italian regions [32] our findings may not reflect paediatric antibiotic prescribing in Italy as a whole and the appropriateness should be investigated in all Italian regions as part of drug utilization monitoring plan. As reported by Cartabia et al. [32], the geographic distribution is not explained by the health status of the children but by the tendency of the pediatrician to prescribe drugs. In addition, we collected data to assess pneumonia severity or other factors that may have influenced decisions regarding diagnostic testing and antibiotic management. Of note that in the first phase of this study, pediatricians were kept unaware of the present evaluation, so their prescribing of antibiotic practices could not have been influenced by the study: therefore our approach permitted objective evaluation of physicians' prescribing habits in everyday practice. Another strength of the present paper is that the study period spanned over one-year to avoid seasonal variation in illness that may affect antibiotic prescribing.

Among limitations, data on microbiological investigation were not available in all cases, therefore some patients diagnosed with pneumonia may have had viral etiologies. However, even with the understanding that some pneumonias in our study population were likely viral, the misdiagnosis of viral pneumonias as bacterial should not have affected antibiotic selection once the physician's intent was to treat bacterial pneumonia.

In the surveillance part of the MAREA study, a needed sample size of 840 patients (420 patients each phase) was calculated assuming an expected frequency of hospitalization of about $10 \%$ in pediatric population with a diagnosis of pneumonia, AOM and/or pharyngotonsillitis. We enrolled only 620 patients but this only slightly decreased the statistical power (from $80 \%$ to $75 \%$ ) because the observed frequency of hospitalization for the three indications was higher than expected $(25 \%$ vs. $10 \%$, data not shown). Alternatively, to determine the hospitalization percentage more accurately, we should have conducted a pilot study. Being the appropriateness study strictly depend on the surveillance study (i.e. the number antibiotics prescriptions are related to the number of patients enrolled), the same sample size was kept also for the appropriateness study.

\section{Conclusion}

Our study shows that prescribing antibiotic practices were globally sufficiently appropriate, but certain aspects deserve closer investigation, above all the widespread preference for multidrug empirical regimens or macrolide in monotherapy which in some cases seemed difficult to justify.

In addition, findings from this study underline that priority actions for improving the rational use of antibacterials in children should concentrate on education activities such as active participation of the pediatricians to training courses or availability of electronic decision support systems that may facilitate guideline-concordant antibiotic prescription. 


\section{Abbreviations}

ADR: Adverse drug reactions; AOM: Acute otitis media; e-CRF: Electronic case report form; El: Enzyme inhibitor; H-CAP: Hospitalized pneumonia; NotHCAP: Not-hospitalized pneumonia; SD: Standard deviation

\begin{abstract}
Acknowledgements
Complete list of The Pediatric Ligurian Network - MAREA network contributors: Roberto Cambiaso (primary care pediatrician - Local Medical Service), Simonetta Cozzani (Pediatric Dpt, Ospedale Sant'Andrea, La Spezia), Anna-Carla Defilippi (Sanremo Hospital- IM), Maria Cristina Diana (Pediatric Emergency Dpt - Istituto G. Gaslini, Genoa), Alberto Ferrando (primary care pediatrician - Local Medical Service), Michele Fiore (primary care pediatrician - Local Medical Service), Donata Girosi (Pediatric Pulmonology and Allergy Unit and Cystic Fibrosis Center - Istituto G. Gaslini, Genoa), Alessandro Insolvibile (Sanremo Hospital- IM), Giorgio Lepre (primary care pediatrician - Local Medical Service), Claudio Muià (primary care pediatrician - Local Medical Service), Gianfranco Picollo (primary care pediatrician - Local Medical Service), Giovanni Semprini (primary care pediatrician - Local Medical Service), Giuseppe Squazzini (primary care pediatrician - Local Medical Service), Laura Tegaldo (Neonatology Unit Ospedale S. Carlo Evangelico - Genoa), Alberto Traverso (Santa Corona HospitalSavona), Barbara Tubino Pediatric Emergency Dpt - Istituto G. Gaslini, Genoa, Giovanna Villa (Pediatric Emergency Dpt - Istituto G. Gaslini, Genoa). This study was funded by Health Regional Agency of the Liguria Region/ Italian Medicines Agency.
\end{abstract}

\section{Funding}

This study was funded by Health Regional Agency of the Liguria Region/ Italian Medicines Agency.

\section{Availability of data and materials}

The MAREA database is not available for free access.

\section{Consent for publication}

NA

\section{Authors' contributions}

PdP conceived and designed the study; supervised the research group; contributed to the interpretation of the data; revised critically the manuscript for important intellectual content; ODCA made substantial contributions to conception, design, planning and acquisition; supervised the research group; revised critically the manuscript for important intellectual content; MS contributed to data management; performed statistical analysis and interpretation of the data; revised the scientific literature; was involved in drafting the manuscript; MAT, AR, GC, GAR, EC, MCM, SZ took part in designing the study and made substantial contributions to analysis and interpretation of data; SR took part in designing the study; contributed to the interpretation of the data; revised the scientific literature; was a major contributor in writing the manuscript; Pediatric Ligurian Network - MAREA network: RC, SC, AD, MCD, AF, MF, DG, Al, GL, CM, GP, GS, GS, LT, AT, BT, GV made substantial contributions to patient enrolment and data management; made their contribution in revising the manuscript. All of the authors read and approved the final version of the manuscript. All the authors agreed to be accountable for all aspects of the work in ensuring that questions related to the accuracy or integrity of any part of the work are appropriately investigated and resolved.

\section{Ethics approval and consent to participate}

The surveillance and the appropriateness studies were approved by Ethics Committee of the Gaslini Institute (PdP_SR_IGG001). For the surveillance study, all participants and their parents or tutors were informed in detail on the experimental procedure and the main scope of the study and provided written informed consent: For the appropriateness study, in the pre-intervention phase, pediatricians were kept unaware of the aim of the study.

\section{Competing interests}

The authors declare that they have no competing interests.

\section{Publisher's Note}

Springer Nature remains neutral with regard to jurisdictional claims in published maps and institutional affiliations.

\section{Author details}

${ }^{1}$ Pediatric Emergency Department, Istituto G, Gaslini, Genoa, Italy. ${ }^{2}$ Clinical Pharmacology \& Clinical Trials Unit, Istituto G, Gaslini, Genoa, Italy. ${ }^{3}$ Pediatric Pulmonology and Allergy Unit and Cystic Fibrosis Center - Istituto G, Gaslini, Genoa, Italy. ${ }^{4}$ Primary Care Pediatrician - FIMP Genoa, Genoa, Italy. ${ }^{5}$ Infectious Disease Unit, Istituto G, Gaslini, Genoa, Italy. ${ }^{6}$ Health Regional Agency of the Liguria Region, Genoa, Italy.

Received: 3 April 2017 Accepted: 30 November 2017

Published online: 22 December 2017

\section{References}

1. Bradley CP. Decision making and prescribing patterns-a literature review. Fam Pract. 1991;8(3):276-87.

2. Jones DA, Sweetnam PM, Elwood PC. Drug prescribing by GPs in wales and in england. J Epidemiol Community Health. 1980 Jun;34(2):119-23.

3. NICE NICE Short Clinical Guidelines Technical Team, 2008.

4. Bronzwaer SL, Cars O, Buchholz U, Molstad S, Goettsch W, Veldhuijzen IK, et al. A european study on the relationship between antimicrobial use and antimicrobial resistance. Emerg Infect Dis. 2002:8(3):278-82.

5. Harris M, Clark J, Coote N, Fletcher P, Harnden A, McKean M, et al. British thoracic society guidelines for the management of community acquired pneumonia in children: update 2011. Thorax. 2011;66(Suppl 2):ii1-23.

6. Weissman J, Besser RE. Promoting appropriate antibiotic use for pediatric patients: a social ecological framework. Semin Pediatr Infect Dis. 2004;15(1):41-51.

7. de Bie S, Kaguelidou F, Verhamme KM, De Ridder M, Picelli G, Straus SM, et al. Using prescription patterns in primary care to derive new quality indicators for childhood community antibiotic prescribing. Pediatr Infect Dis 2016:35(12):1317-23.

8. Esposito S, Cohen R, Domingo JD, Pecurariu OF, Greenberg D, Heininger U, et al. Antibiotic therapy for pediatric community-acquired pneumonia: do we know when, what and for how long to treat? Pediatr Infect Dis J. 2012; 31(6):e78-85.

9. Costelloe C, Metcalfe C, Lovering A, Mant D, Hay AD. Effect of antibiotic prescribing in primary care on antimicrobial resistance in individual patients: systematic review and meta-analysis. BMJ. 2010;340:c2096.

10. van de Sande-Bruinsma N, Grundmann H, Verloo D, Tiemersma E, Monen J, Goossens $\mathrm{H}$, et al. Antimicrobial drug use and resistance in europe. Emerg Infect Dis. 2008;14(11):1722-30.

11. Andrews T, Thompson M, Buckley DI, Heneghan C, Deyo R, Redmond N, et al. Interventions to influence consulting and antibiotic use for acute respiratory tract infections in children: a systematic review and metaanalysis. PLoS One. 2012;7(1):e30334.

12. Perz JF, Craig AS, Coffey CS, Jorgensen DM, Mitchel E, Hall S, et al. Changes in antibiotic prescribing for children after a community-wide campaign. JAMA. 2002;287(23):3103-9.

13. Piovani D, Clavenna A, Sequi M, Cartabia M, Bortolotti A, Fortino I, et al. Reducing the costs of paediatric antibiotic prescribing in the community by implementing guideline recommendations. J Clin Pharm Ther. 2013;38(5): 373-8.

14. Weddle G, Goldman J, Myers A, Newland J. Impact of an educational intervention to improve antibiotic prescribing for nurse practitioners in a pediatric urgent care center. J Pediatr Health Care. 2017;31(2):184-8.

15. Ciofi degli Atti ML, Massari M, Bella A, Boccia D, Filia A, Salmaso S, et al. Clinical, social and relational determinants of paediatric ambulatory drug prescriptions due to respiratory tract infections in italy. Eur J Clin Pharmacol. 2006:62(12):1055-64

16. Nash DR, Harman J, Wald ER, Kelleher KJ. Antibiotic prescribing by primary care physicians for children with upper respiratory tract infections. Arch Pediatr Adolesc Med. 2002;156(11):1114-9.

17. Boonstra $\mathrm{E}$, Lindbaek $\mathrm{M}, \mathrm{Ngome} \mathrm{E}$. Adherence to management guidelines in acute respiratory infections and diarrhoea in children under 5 years old in primary health care in botswana. Int J Qual Health Care. 2005;17(3):221-7.

18. Hamm RM, Hicks RJ, Bemben DA. Antibiotics and respiratory infections: are patients more satisfied when expectations are met? J Fam Pract. 1996; 43(1):56-62.

19. Mainous AG 3rd, Hueston WJ, Love MM. Antibiotics for colds in children: who are the high prescribers? Arch Pediatr Adolesc Med. 1998;152(4):349-52.

20. Mainous AG 3rd, Zoorob RJ, Oler MJ, Haynes DM. Patient knowledge of upper respiratory infections: implications for antibiotic expectations and unnecessary utilization. J Fam Pract. 1997;45(1):75-83. 
21. Cabana MD, Rand CS, Powe NR, Wu AW, Wilson MH, Abboud PA, et al. Why don't physicians follow clinical practice guidelines? A framework for improvement. JAMA. 1999;282(15):1458-65.

22. WHO. The pursuit of responsible use of medicines: sharing and learning from country experiences. Available from: http://www.who.int/medicines/ publications/responsible_use/en/. Accessed 7 Feb 2017.

23. Ciofi degli Atti M, Spila Alegiani S, Raschetti R, Arace P, Giusti A, Spiazzi R, et al. Surgical antibiotic prophylaxis in children: adherence to indication, choice of agent, timing, and duration. Eur J Clin Pharmacol. 2015;71(4):483-8.

24. Hodgson KA, Huynh J, Ibrahim LF, Sacks B, Golshevsky D, Layley M, et al. The use, appropriateness and outcomes of outpatient parenteral antimicrobial therapy. Arch Dis Child. 2016;101(10):886-93.

25. Mahdaviazad H, Masoompour SM, Askarian M. Iranian surgeons' compliance with the american society of health-system pharmacists guidelines: antibiotic prophylaxis in private versus teaching hospitals in shiraz, iran. J Infect Public Health. 2011;4(5-6):253-9.

26. Westphal JF, Jehl F, Javelot H, Nonnenmacher C. Enhanced physician adherence to antibiotic use guidelines through increased availability of guidelines at the time of drug ordering in hospital setting. Pharmacoepidemiol Drug Saf. 2011;20(2):162-8.

27. Saleh EA, Schroeder DR, Hanson AC, Banerjee R. Guideline-concordant antibiotic prescribing for pediatric outpatients with otitis media, community-acquired pneumonia, and skin and soft tissue infections in a large multispecialty healthcare system. Clin Res Infect Dis. 2015;2(1):1010

28. Kronman MP, Hersh AL, Feng R, Huang YS, Lee GE, Shah SS. Ambulatory visit rates and antibiotic prescribing for children with pneumonia, 19942007. Pediatrics. 2011;127(3):411-8

29. Boccia D, Alegiani SS, Pantosti A, Moro ML, Traversa G. The geographic relationship between the use of antimicrobial drugs and the pattern of resistance for streptococcus pneumoniae in italy. Eur J Clin Pharmacol. 2004; 60(2):115-9.

30. Seppala H, Klaukka T, Vuopio-Varkila J, Muotiala A, Helenius H, Lager K, et al. The effect of changes in the consumption of macrolide antibiotics on erythromycin resistance in group a streptococci in finland. Finnish study group for antimicrobial resistance. N Engl J Med. 1997;337(7):441-6.

31. European Centre for Disease Prevention and Control. Antimicrobial resistance surveillance in Europe 2014. Annual report of the European antimicrobial resistance surveillance network (EARS-net). Stockholm: ECDC; 2015.

32. Cartabia M, Campi R, Clavenna A, Bortolotti A, Fortino I, Merlino L, et al. Geographical epidemiology of antibacterials in the preschool age. Int J Health Geogr. 2012;11. https://doi.org/10.1186/1476-072X-11-52

33. Clavenna A, Bonati M. Differences in antibiotic prescribing in paediatric outpatients. Arch Dis Child. 2011;96(6):590-5.

34. De Luca M, Dona D, Montagnani C, Lo Vecchio A, Romanengo M, Tagliabue $\mathrm{C}$, et al. Antibiotic prescriptions and prophylaxis in italian children. Is it time to change? Data from the ARPEC project. PLoS One. 2016;11(5):e0154662.

35. Principi N, Esposito S. Emerging role of mycoplasma pneumoniae and chlamydia pneumoniae in paediatric respiratory-tract infections. Lancet Infect Dis. 2001;1(5):334-44.

36. Esposito S, Blasi F, Allegra L, Principi N, Mowgli Study Group. Use of antimicrobial agents for community-acquired lower respiratory tract infections in hospitalised children. Eur J Clin Microbiol Infect Dis. 2001;20(9):647-50.

37. Bradley JS, Byington CL, Shah SS, Alverson B, Carter ER, Harrison C, et al. The management of community-acquired pneumonia in infants and children older than 3 months of age: clinical practice guidelines by the Pediatric Infectious Diseases Society and the Infectious Diseases Society of America. Clin Infect Dis. 2011;53(7):e25-76.

38. Rachina S, Kozlov R, Belkova $\mathrm{W}$, Tatochenko G and PATRIOT study group. Paediatricians' approach to prescribing of systemic antibiotics in outpatient children with respiratory infections in Russia. $25^{\text {th }}$ European Congress. 2015: ECCMID, 25-28 May 2015. Available from: https://www.escmid.org/escmid_ publications/escmid_elibrary/?q=Paediatricians\%E2\%80\%99+approach+to +prescribing+of+systemic+antibiotics+in+outpatient+children+with+respiratory +infections+in+Russia\&id $=2173 \& \mathrm{~L}=0 \& \mathrm{x}=0 \& \mathrm{y}=0 \& \mathrm{tx}$ _solr\%5Bfilter\%5D\%5B1\%5D= author\%253AYuliya\%2BBelkova\&tx_solr\%5Bfilter\%5D\%5B2\%5D=main_filter_ eccmid\%253Atrue. Accessed 11 Oct 2017.
39. Neuman MI, Hall M, Hersh AL, Brogan TV, Parikh K, Newland JG, et al. Influence of hospital guidelines on management of children hospitalized with pneumonia. Pediatrics. 2012;130(5):e823-30.

40. Pellegrino P, Carnovale C, Cattaneo D, Perrone V, Antoniazzi S, Pozzi M, et al. Pharmacovigilance knowledge in family paediatricians. A survey study in italy. Health Policy. 2013;113(1-2):216-20.

\section{Submit your next manuscript to BioMed Central and we will help you at every step:}

- We accept pre-submission inquiries

- Our selector tool helps you to find the most relevant journal

- We provide round the clock customer support

- Convenient online submission

- Thorough peer review

- Inclusion in PubMed and all major indexing services

- Maximum visibility for your research

Submit your manuscript at www.biomedcentral.com/submit 